\title{
ROCK Inhibitor Converts Corneal Endothelial Cells into a Phenotype Capable of Regenerating In Vivo Endothelial Tissue
}

\author{
Naoki Okumura, ${ }^{\star \dagger}$ Noriko Koizumi, ${ }^{\dagger}$ Morio Ueno, ${ }^{*}$ \\ Yuji Sakamoto, ${ }^{\dagger}$ Hiroaki Takahashi, ${ }^{\dagger}$ \\ Hideaki Tsuchiya, ${ }^{\ddagger}$ Junji Hamuro, ${ }^{*}$ and \\ Shigeru Kinoshita*

\begin{abstract}
From the Department of Ophthalmology,* Kyoto Prefectural University of Medicine, Kyoto; the Department of Biomedical Engineering, ${ }^{\dagger}$ Faculty of Life and Medical Sciences, Doshisha University, Kyotanabe; and the Research Center for Animal Life Science, ${ }^{\ddagger}$ Shiga University of Medical Science, Otsu, Japan
\end{abstract}

Corneal endothelial dysfunction accompanied by visual disturbance is a primary indication for corneal transplantation. We previously reported that the adhesion of corneal endothelial cells (CECs) to a substrate was enhanced by the selective ROCK inhibitor Y-27632. It is hypothesized that the inhibition of ROCK signaling may manipulate cell adhesion properties, thus enabling the transplantation of cultivated CECs as a form of regenerative medicine. In the present study, using a rabbit corneal endothelial dysfunction model, the transplantation of CECs in combination with Y-27632 successfully achieved the recovery of corneal transparency. Complications related to cell injection therapy, such as the abnormal deposition of the injected cells as well as the elevation of intraocular pressure, were not observed. Reconstructed corneal endothelium with Y-27632 exhibited a monolayer hexagonal cell shape with a normal expression of function-related markers, such as $\mathrm{ZO}-1$, and $\mathrm{Na}^{+}$/ $\mathrm{K}^{+}$-ATPase, whereas reconstruction without Y-27632 exhibited a stratified fibroblastic phenotype without the expression of markers. Moreover, transplantation of CECs in primates in the presence of the ROCK inhibitor also achieved the recovery of long-term corneal transparency with a monolayer hexagonal cell phenotype at a high cell density. Taken together, these results suggest that the selective ROCK inhibitor Y-27632 enables cultivated CEC-based therapy and that the modulation of Rho-ROCK signaling activity serves to enhance cell engraftment for cell-based re- generative medicine. (Am J Pathol 2012, 181:268-277; bttp://dx.doi.org/10.1016/j.ajpath.2012.03.033)

Corneal endothelial dysfunction is a major cause of severe visual impairment, because corneal endothelial cells maintain corneal transparency through their barrier and $\mathrm{Na}^{+}-\mathrm{K}^{+}$transport system. Highly effective surgical techniques to replace corneal endothelium (eg, Descemet's stripping endothelial keratoplasty) have been developed, ${ }^{1,2}$ aimed at replacing penetrating keratoplasty for overcoming pathological dysfunctions of corneal endothelial tissue. Several research groups, including ours, have devoted an intensive amount of effort in an attempt to establish new treatment methods suitable for a practical clinical intervention to repair corneal endothelial dysfunctions. ${ }^{3-6}$ Because corneal endothelium is composed of a monolayer and is technically difficult to transplant into the anterior chamber as a structurally flexible cell sheet, those research teams cultured corneal endothelial cells (CECs) on substrates such as collagen sheets and amniotic membrane.

The injection of cultivated cells has been reported for the treatment of a number of organs associated with degenerative diseases such as the heart, ${ }^{7}$ vessels, ${ }^{8}$ pancreas, ${ }^{9}$ and cartilage. ${ }^{10}$ In regard to corneal endothelium, it is known that injected cultured CECs appear to be washed off by aqueous humor flow, thus resulting in the poor adhesion of those injected cells onto the corneal tissue. To develop an effective method for delivering cultivated CECs to the posterior cornea, the magnetic attachment of iron powder or superparamagnetic microspheres incorporated in the cultivated CECs has been attempted. This method has been shown to work in a

Supported in part by the Highway Program for realization of regenerative medicine (S.K. and N.O.) and the Funding Program for Next Generation World-Leading Researchers from the Cabinet Office in Japan (LS117 to N.K.).

Accepted for publication March 27, 2012.

Address reprint requests to Noriko Koizumi, M.D., Ph.D., Department of Biomedical Engineering, Faculty of Life and Medical Sciences, DoshishaUniversity, Kyotanabe610-0321, Japan.E-mail:nkoizumi@mail. doshisha.ac.jp. 
rabbit transplantation model ${ }^{11,12}$ and in an organ culture model of the human eye ${ }^{13}$; however, these methods have yet to be applied in the clinical setting.

Cell adhesion is known to be mediated through transmembrane adhesion molecules linked to the intracellular cytoskeleton. In addition to the structural function, these adhesion molecules reportedly serve as a platform for the interplay with the surrounding environments. ${ }^{14,15}$ Rho GTPase proteins are key modulators of cytoskeletal dynamics that occur after cell adhesion. ${ }^{16-18}$ It has been reported that Rho GTPases induce a specific type of actin cytoskeleton through mediating downstream effectors $\mathrm{mDia}$ and Rho-associated kinase (ROCK), and that they regulate a variety of cellular functions. ${ }^{19}$ Cell adhesion, motility, and cell morphogenesis are thought to be determined by the balance between $\mathrm{mDia}$ and ROCK activities. ${ }^{19}$ We recently reported that the adhesion of CECs to a substrate was enhanced by inhibiting Rho/ ROCK signaling. ${ }^{20}$ This finding coincides well with those of other studies that demonstrated that Rho-ROCK signaling negatively regulates the integrin-mediated adhesion of monocytes, and that the inhibition of ROCK by a selective ROCK inhibitor upregulates adhesion. ${ }^{17,18}$ These features have led us to hypothesize that the inhibition of ROCK signaling may provide a way to manipulate the cell adhesion property of cultivated corneal endothelium to the extent practical for regenerative medicine.

In this current study, in two animal models (rabbit and primate) of corneal endothelial dysfunctions, the transplantation of cultivated CECs in combination with ROCK inhibitor Y-27632 successfully achieved the recovery of corneal transparency. Inhibition of the ROCK signaling manipulated the adhesion property of the cultivated CECs. Moreover, the injected CECs functioned sufficiently well to reconstruct the corneal endothelium with an appropriate cell density, morphology, and expression of function-related markers. This novel treatment strategy may provide a new therapeutic modality for corneal-endothelium-associated pathological dysfunctions.

\section{Materials and Methods}

\section{Materials}

Rabbit eyes were purchased from Funakoshi Corporation (Tokyo, Japan). Alizarin red S stain and selective ROCK inhibitor Y-27632 were purchased from Wako Pure Chemical Industries, Ltd. (Osaka, Japan). Dulbecco's modified Eagle's medium supplemented with penicillin, streptomycin, and basic fibroblast growth factor (bFGF), Vybrant Dil cell-labeling solution, Alexa Fluor 594-conjugated phalloidin, Alexa Fluor 488-conjugated phalloidin, Alexa Fluor 488-conjugated goat anti-mouse IgG, antivinculin antibody, ROCK1 Stealth RNAi (HSS109291, HSS109292, and HSS109293), ROCK2 Stealth RNAi (HSS114106, HSS114107, and HSS114108), Stealth RNAi negative control medium GC \#2, and Lipofectamine RNAiMAX were purchased from Life Technologies (Carlsbad, CA). Dispase II was purchased from Roche Applied Science (Penzberg, Germany). FNC Coating Mix was purchased from Athena Environmental Sciences, Inc. (Baltimore, MD). Ki-67 monoclonal antibody, propidium iodide (PI), and Cytochalasin D were purchased from Sigma-Aldrich Co. (St. Louis, MO). ZO-1 polyclonal antibody was purchased from Zymed Laboratories (South San Francisco, CA). $\alpha$-Smooth muscle actin ( $\alpha$-SMA) monoclonal antibody was purchased from Thermo Fisher Scientific (Kalamazoo, MI). $\mathrm{Na}^{+} / \mathrm{K}^{+}$-ATPase monoclonal antibody was purchased from Upstate Biotech (Lake Placid, NY). DAPI was purchased from Vector Laboratories (Burlingame, CA). CellTiter-Glo Luminescent Cell Viability Assay was purchased from Promega (Madison, WI).

\section{Animal Experiment Approval}

In all experiments, animals were housed and treated in accordance with The Association for Research in Vision and Ophthalmology Statement for the Use of Animals in Ophthalmic and Vision Research. The rabbit experiments were performed at Doshisha University (Kyoto, Japan) according to the protocol approved by that university's Animal Care and Use Committee (approval no. 0831). The monkey experiments were performed at the Research Center for Animal Life Science at Shiga University of Medical Science (Otsu, Japan) according to the protocol approved by that university's Animal Care and Use Committee (approval no. 2008-10-5).

\section{Cell Culture of Rabbit and Monkey CECs}

Ten rabbit eyes were used for the rabbit CECs (RCECs) culture. Eight corneas from four cynomolgus monkeys ( 3 to 5 years of age; estimated equivalent human age: 5 to 20 years) housed at the Nissei Bilis Co. (Otsu, Japan) and the Keari Co. (Wakayama, Japan), respectively, were used for the monkey CECs (MCECs) culture. The RCECs and MCECs were cultivated as described previously. ${ }^{3,20}$ Briefly, Descemet's membrane with CECs was stripped and incubated in $0.6 \mathrm{U} / \mathrm{mL}$ of Dispase II to release the CECs. After a 60 -minute incubation at $37^{\circ} \mathrm{C}$, the CECs obtained from individual corneas were resuspended in culture medium and plated in one well of a six-well plate coated with cell attachment reagent (FNC Coating Mix). All primary cell cultures and serial passages of CECs were performed in growth medium composed of Dulbecco's modified Eagle's medium supplemented with 10\% fetal bovine serum, $50 \mathrm{U} / \mathrm{mL}$ penicillin, $50 \mu \mathrm{g} / \mathrm{mL}$ streptomycin, and $2 \mathrm{ng} / \mathrm{mL}$ bFGF. CECs were cultured in a humidified atmosphere at $37^{\circ} \mathrm{C}$ in $5 \% \mathrm{CO}_{2}$. The culture medium was changed every 2 days. When cells reached confluency in 10 to 14 days, they were rinsed in $\mathrm{Ca}^{2+}$ and $\mathrm{Mg}^{2+}$-free Dulbecco's phosphate-buffered saline (PBS), trypsinized with $0.05 \%$ Trypsin-EDTA (Life Technologies, Carlsbad, CA) for 5 minutes at $37^{\circ} \mathrm{C}$, and passaged at ratios of $1: 2$ to $1: 4$. Cultivated CECs derived from both rabbit and monkey corneas at passages 3 through 5 were used for all experiments. To confirm the cultivation of the CECs, the morphology and density of the cultivated cells were compared with normal in vivo rabbit CECs examined using a noncontact specular microscope (Noncon Robo, SP-8800; Konan Medical, Nishinomiya, 
Japan) and stained with Alizarin red. In some experiments, to investigate the fate of the injected CECs in vivo, the CECs were labeled with fluorescein by use of the Vybrant Dil cell-labeling solution according to the manufacturer's protocol.

\section{Rabbit Corneal Endothelial Dysfunction Model}

To create rabbit corneal endothelial pathological dysfunction models, the lenses of both eyes of 12 Japanese white rabbits were removed under general anesthesia by use of the Alcon Series 20000 Legacy Surgical System (Alcon, Fort Worth, TX) to deepen the anterior chamber. Next, the corneal endothelium of each of those eyes was mechanically scraped with a 20-gauge silicone needle (Soft Tapered Needle; Inami, Tokyo, Japan) from Descemet's membrane as described previously. ${ }^{3,4}$ The scraped area was then confirmed by $0.04 \%$ trypan blue staining during surgery. In the preliminary experiments, we confirmed that Descemet's membrane was intact, the mechanically scraped area had no cells on Descemet's membrane, and that residual CECs were detected in only a 500- to 600- $\mu \mathrm{m}$ area at the edge of Descemet's membrane.

\section{Injection of Cultivated CECs into the Rabbit Eyes}

To evaluate the injection of cultivated CECs with ROCK inhibitor, RCECs at a density of $2.0 \times 10^{5}$ cells were suspended in $200 \mu \mathrm{l}$ DMEM supplemented with $100 \mu \mathrm{mol} / \mathrm{L}$ of Y-27632 and then injected into the anterior chamber of the eyes of the above-described corneal endothelial dysfunction rabbit model. RCECs with Y-27632 were injected into the right eyes of six rabbits, and RCECs without Y-27632 were injected into the right eyes of the other six rabbits. After the injection, the eyes of those 12 rabbits were kept in the face-down position for 3 hours under general anesthesia. The left eyes of those 12 rabbits in which the corneal endothelium was removed mechanically were used as a control. One rabbit injected with RCECs with Y-27632 and one rabbit injected with RCECs without Y-27632 were euthanized 3 hours after injection for histological examination. The corneal appearance of the other 10 rabbits was examined daily by use of a slit-lamp microscope for the first week, and then once every 2 days for the following 2 weeks. Those 10 rabbits were then euthanized for histological examination. Corneal thickness was determined by use of an ultrasound pachymeter (SP2000; Tomey, Nagoya, Japan), and the mean of 10 measured values was then calculated (up to a maximum thickness of $1200 \mu \mathrm{m}$, the instrument's maximum reading). Intraocular pressure was measured by use of a pneumatonometer (30 Classic; Reichert, NY).

\section{Histological Examination of Rabbit Eyes After CEC Injection}

Sections (6- $\mu \mathrm{m})$ of corneal specimens obtained from the 10 rabbits euthanized 2 weeks after injection were embedded in OCT compound and then fixed in $4 \%$ formaldehyde. Differential interference contrast (DIC) images and fluorescence images of Dil-labeled cells were obtained by use of a fluorescence microscope (TCS SP2 AOBS; Leica Microsystems, Welzlar, Germany). For flatmount examinations, whole corneal specimens were fixed in $4 \%$ formaldehyde and incubated in $1 \%$ bovine serum albumin (BSA) to block any nonspecific binding. To evaluate the effect of $\mathrm{Y}-27632$ on the adhesion property of the cells, corneas obtained from the 2 rabbits euthanized 3 hours after injection were examined by actin staining performed with a 1:400 dilution of Alexa Fluor 488-conjugated phalloidin. Actin staining was used to evaluate the cellular morphology. The cell nuclei were then stained with PI. To investigate the phenotype of the reconstructed corneal endothelium obtained from the 10 rabbits euthanized 2 weeks after injection, immunohistochemical analyses of actin, $\alpha$-SMA, ZO- $1, \mathrm{Na}^{+} / \mathrm{K}^{+}$-ATPase, Dil, and Ki-67 were performed. $\alpha$-SMA was used to evaluate the fibroblastic change. ZO-1, a tight-junction-associated protein, and $\mathrm{Na}^{+} / \mathrm{K}^{+}$-ATPase, the protein associated with pump function, were used for function related markers of CECs. The $\alpha$-SMA, ZO- 1 , and $\mathrm{Na}^{+} / \mathrm{K}^{+}$-ATPase staining were performed with a 1:200 dilution of $\alpha$-SMA monoclonal antibody, ZO-1 polyclonal antibody, and $\mathrm{Na}^{+} / \mathrm{K}^{+}$-ATPase monoclonal antibody, respectively. Ki-67 (a cell-proliferation-related maker) staining was performed using a 1:400 dilution of anti-mouse Ki-67 antibody. For the secondary antibody, a 1:2000 dilution of Alexa Fluor 488-conjugated goat anti-mouse IgG was used. Cell nuclei were then stained with DAPI, and the slides were inspected by fluorescence microscopy.

\section{Effect of Y-27632 on MCECs in Culture}

MCECs were cultured at a density of $2.5 \times 10^{4} \mathrm{cells} / \mathrm{cm}^{2}$ on Lab-Tek Chamber Slides (NUNC A/S, Roskilde, Denmark). Actin staining was performed with 1:400-diluted Alexa Fluor, as described above, after 24 hours of seeding, and vinculin staining was performed using 1:200diluted vinculin after 3 hours of seeding. The number of attached MCECs was evaluated by use of CellTiter-Glo Luminescent Cell Viability Assay performed according to the manufacturer's protocol. The MCECs were seeded with a different concentration of Y-27632 at the density of $1.0 \times 10^{3}$ cells onto 96-well plates, and the number of adhered MCECs at 24 hours after seeding was then measured by use of a Veritas Microplate Luminometer (Promega). In addition to ROCK signaling inhibition, to evaluate the effect of inhibition of actin polymerization on CECs adhesion, MCECs were seeded with a different concentration of cytochalasin $\mathrm{D}$ at the density of $1.0 \times$ $10^{3}$ cells onto 96-well plates, and the number of adhered MCECs at 24 hours after seeding was then measured. Five samples were prepared for each group.

To determine the adhesion property of the MCECs onto the basement membrane, the cells were seeded onto rabbit corneas in which the corneal endothelium was mechanically denuded and the basement membranes were exposed. The cells were seeded at the density of $2.5 \times 10^{4}$ cells $/ \mathrm{cm}^{2}$ suspended in culture medium supplemented with or without $10 \mu \mathrm{mol} / \mathrm{L}$ Y-27632. Actin staining was performed at 3 hours after seeding in the same 
manner as with the Alexa Fluor 488-conjugated phalloidin staining described above. Cell nuclei were then stained with PI. MCECs at the density of $2.0 \times 10^{5}$ cells were also seeded, with or without Y-27632, onto Descemet's membrane of four rabbits from each group, and the membrane was then mechanically peeled off at 3 hours after seeding. The adhered MCECs were recovered by trypsin digestion, and the cell numbers were then counted.

\section{Inhibition of ROCK Signaling by siRNA on MCECs in Culture}

MCECs seeded at the density of $2.5 \times 10^{4}$ cells $/ \mathrm{cm}^{2}$ onto a 24-well plate were incubated with RNAi duplex (ROCK1 Stealth RNAi and ROCK2 Stealth RNAi) and Lipofectamine RNAiMAX according to the manufacturer's protocol. Briefly, 1 day before transfection, the culture medium was replaced with fresh medium without antibiotics. RNAi duplex at the final concentration of $10 \mathrm{nmol} / \mathrm{L}$ and Lipofectamine RNAiMAX complexes were added to each well. The MCECs were incubated for 12 hours at $37^{\circ} \mathrm{C}$ in a $\mathrm{CO}_{2}$ incubator. Random RNAi was used as a control. The MCECs were then seeded at the density of $1.0 \times 10^{3}$ cells onto 96-well plates, and the number of attached MCECs was evaluated by use of CellTiter-Glo Luminescent Cell Viability Assay. Knockdown of both ROCK1 and ROCK2, two ROCK isoforms that were identified in the mammalian system, ${ }^{21}$ was confirmed by quantitative PCR analysis (data not shown). Representative data were from six independent experiments using three kinds of ROCK1 Stealth RNAi and ROCK2 Stealth RNAi, respectively.

\section{Injection of Cultivated CECs into Monkey Eyes with Corneal Endothelial Dysfunction}

To create monkey corneal endothelial pathological dysfunction models, the corneal endothelium of the left eyes of four monkeys was mechanically scraped with a 20gauge silicone needle under general anesthesia, as described above for the rabbit model. Next, a $2.0 \times 10^{5}$ density of cultivated MCECs suspended in $200 \mu$ I DMEM supplemented with $100 \mu \mathrm{mol} / \mathrm{L}$ Y-27632 were injected into the anterior chamber of two of the four monkeys. Cultivated MCECs suspended in $200 \mu$ l DMEM without Y-27632 were injected into the anterior chamber of the other 2 monkeys. The eyes of all four monkeys were kept in the face-down position for 3 hours under general anesthesia. The MCECs were labeled with Dil before transplantation. ${ }^{3,4}$ The corneal appearance of all four monkeys was examined daily by use of a slit-lamp microscope for the first week, and then once per week for the following 3 months. Two monkeys from each group (the MCEC-injection with Y-27632 group, and the MCEC-injection without $\mathrm{Y}-27632$ group) were euthanized at 14 days after the injection, and the other 2 monkeys were euthanized at 3 months after the injection. For flat-mount examinations, whole corneal specimens were fixed in $4 \%$ formaldehyde, incubated in 1\% BSA to block nonspecific binding, and then prepared for histological examination. To inves- tigate the phenotype of the reconstructed corneal endothelium, immunohistochemical analyses of actin, ZO-1, and $\mathrm{Na}^{+} / \mathrm{K}^{+}$-ATPase were performed in the same manner as that of the above-described rabbit experiments. After the actin immunostaining, the corneal endothelium of the four monkeys was evaluated by KSS-400EB software version 2.71 (Konan Medical, Hyogo, Japan).

\section{Statistical Analysis}

The statistical significance ( $P$ value) in mean values of the two-sample comparison was determined by Student's $t$-test. Values shown on the graphs represent the mean \pm SEM.

\section{Results}

\section{Injection of Cultivated RCECs with ROCK Inhibitor Enables Regeneration of Cornea in Rabbit Corneal Endothelial Dysfunction Model}

The third-passaged RCECs exhibited a monolayer of hexagonal shaped cells, similar to in vivo RCECs with a cell density of approximately 2600 cells $/ \mathrm{mm}^{2}$ as previously reported ${ }^{5,6}$ (Figure 1A). Cultivated RCECs injected together with Y-27632 were successful in recovering complete transparency of the corneas with pathological dysfunctions. In contrast, RCECs injected without Y-27632 induced hazy and severely edematous corneas, thus indicating that the corneal endothelial dysfunctions were sustained, comparable with those of the control corneas. Slit-lamp microscopy performed at 48 hours after injection revealed complete corneal transparency with the iris and the pupil clearly observed in the eyes injected with RCECs with Y-27632, whereas the iris and pupil could not be observed in the eyes injected with RCECs without Y-27632 and in the control eyes in which the corneal endothelium was mechanically scraped (Figure 1B). Consistent with the slit-lamp microscopy findings, histological analysis performed at 14 days after injection also revealed that the eyes injected with RCECs with Y-27632 had a normal range of corneal thickness, whereas those without Y-27632 exhibited a thick cornea with severe stromal edema. The corneal thicknesses of those specimens were $409 \mu \mathrm{m}$ and $730 \mu \mathrm{m}$, respectively (Figure 1C). In the eyes injected with RCECs with Y-27632, the corneal edema was moderate $(<800 \mu \mathrm{m})$ at day 1 , yet gradually recovered to the normal level. In contrast, in both the control eyes and the eyes injected with RCECs without $Y-27632$, prominent corneal edema $(>1200 \mu \mathrm{m})$ was observed at day 1 , and corneal edema persisted throughout the observation period (Figure 1D). Next, possible complications associated with cell injection into the anterior chamber were investigated, as the injected cells might possibly interfere with normal aqueous humor outflow and produce an increase in intraocular pressure. No abnormal deposition of the injected Dilpositive RCECs onto the trabecular meshwork or onto the iris and no anatomical abnormality such as mechanical angle closure or peripheral anterior synechia were de- 

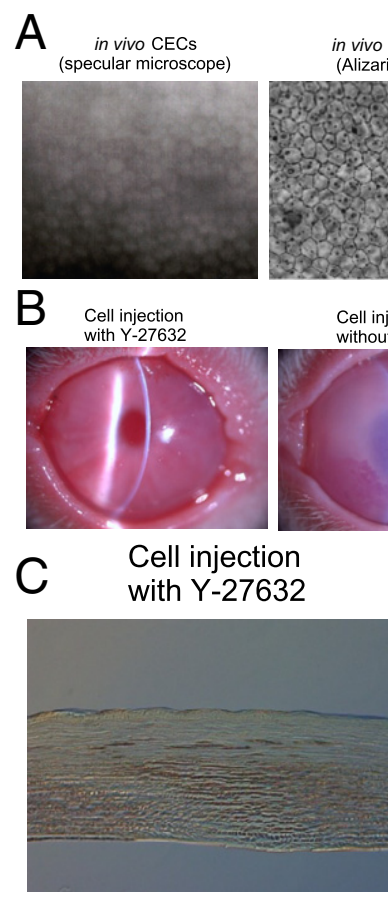

Cell injection
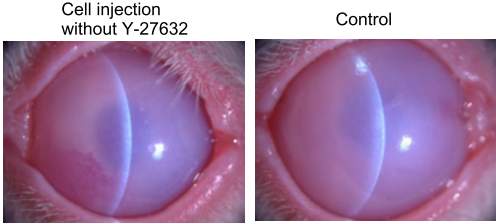

Cell injection without Y-27632
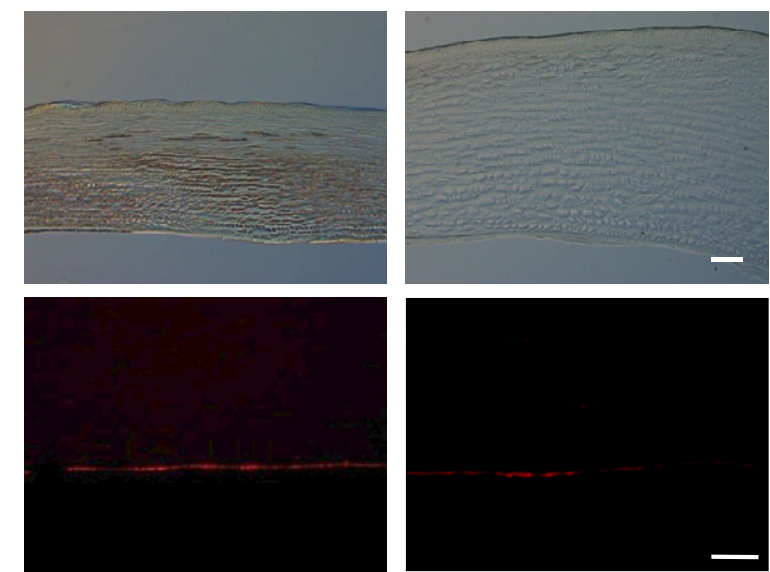

D

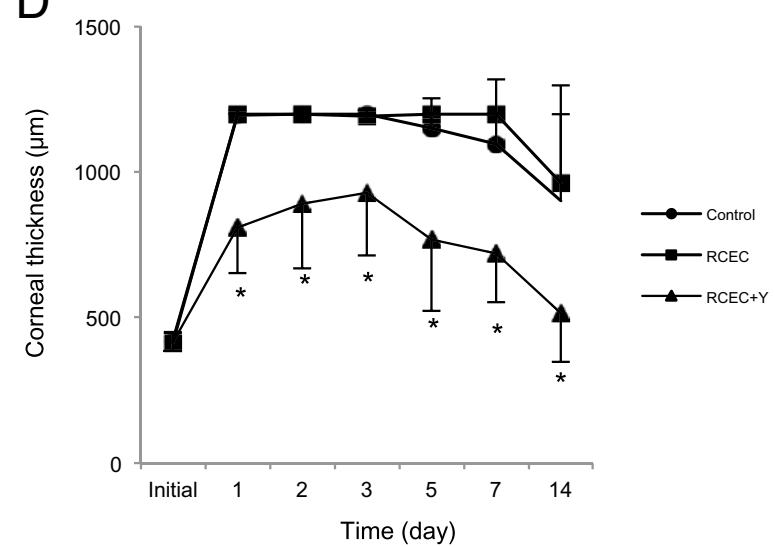

Figure 1. Cell regeneration in a rabbit corneal endothelial dysfunction model A: In vivo normal corneal endothelium (left panel: specular microscope, middle panel: Alizarin red staining) and cultivated rabbit corneal endothelial cells (RCECs) (right panel: phase contrast image). The cultivated RCECs exhibit a homogeneous monolayer of hexagonal cells with a cell density of approximately 2600 cells $/ \mathrm{mm}^{2}$. The morphology and density of the cultivated RCECs are similar to that of in vivo corneal endothelium. Scale bar $=100 \mu \mathrm{m}$. B: Slit-lamp photographs of rabbit eyes injected with cultivated RCECs with Y-27632, cultivated RCECs without Y-27632, and control corneal endothelial dysfunction model after 48 hours. C: Histological analysis of rabbit corneas injected with cultivated RCECs with (left column) or without (right column) Y-27632 (top row: DIC; bottom row: DiI). Injection of RCECs with Y-27632 induces a normal-range thickness $(409 \mu \mathrm{m})$ of the cornea, whereas injection of RCECs without Y-27632 exhibits a thick $(730 \mu \mathrm{m})$ cornea with severe corneal stromal edema at 14 days after injection. Scale bars: $100 \mu \mathrm{m}$. D: Time course of corneal thickness measured by ultrasound pachymeter. In control eyes and in the eyes injected with RCECs without Y-27632, the corneal edema is prominent $(>1200$ $\mu \mathrm{m})$ at day 1 and persists throughout the observation period. In contrast, in the eyes injected with RCECs with Y-27632, the corneal edema is moderate $(<800$ $\mu \mathrm{m})$ at day 1 and gradually recovers to the normal level. tected (Figure 2A). Intraocular pressures were found to be in the normal range in all groups (Figure 2B). To evaluate the injected CECs proliferation status in vivo, a flat-mount cornea was examined at 14 days after injection. Immunofluorescence analysis using the Ki-67 monoclonal antibody (a marker of cell proliferation) revealed that the cell cycle of the nearly all of the injected cells was arrested 2 weeks after injection (Figure 2C). These results

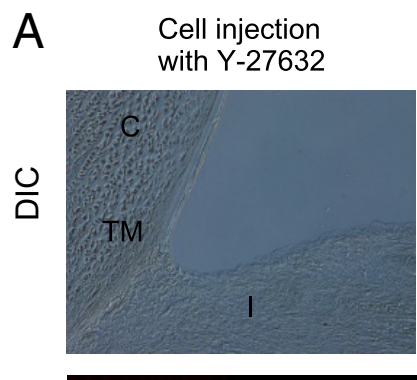
without Y-27632
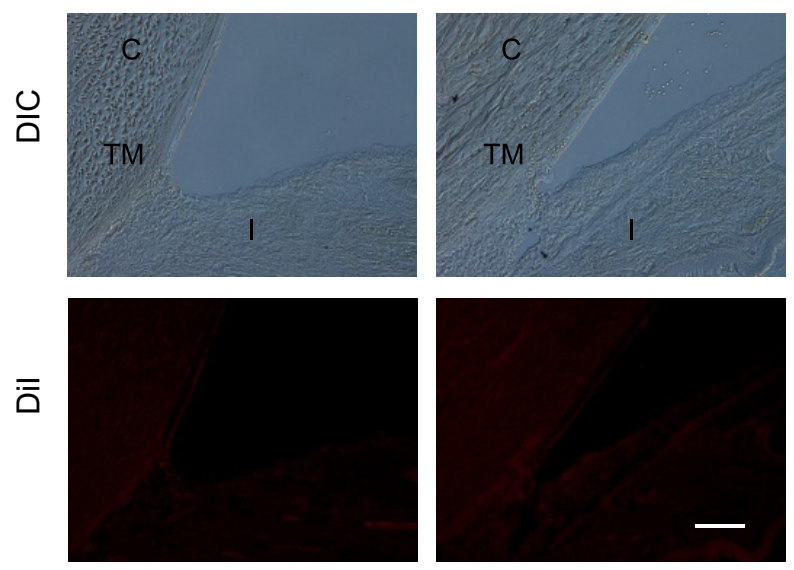

B

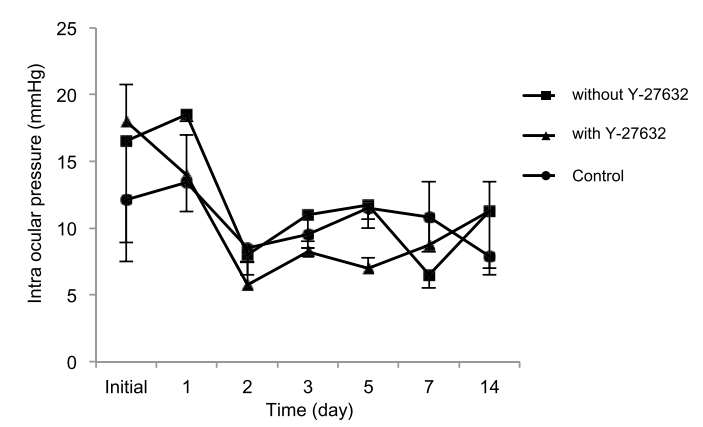

C

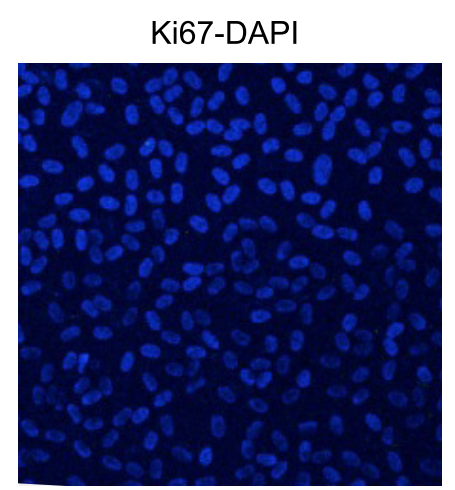

Figure 2. Evaluation of the possible adverse effects of cultivated RCEC injection. A: Histological examination of the iris and the angle tissue. Top row: representative images of the DIC section taken from the rabbit eye injected with cultivated RCECs with or without Y-27632 after 14 days. Bottom row: DiI images of the same sections shown in the top row. C, cornea; I, iris; TM, trabecular meshwork. Scale bar $=100 \mu \mathrm{m}$. B: Intraocular pressures after the injection of RCECs. C: Immunohistochemical staining for cell proliferation marker Ki-67 in the reconstructed RCECs in the eye injected with RCECs with Y-27632 on day 14. 
indicate that ROCK inhibitor can be safely applied for cell injection therapy.

\section{ROCK Inhibitor Provides RCECs with}

\section{Phenotype to Reconstruct Corneal Endothelium}

At 3 hours after injection, RCECs injected with Y-27632 were found to be markedly adhered to the basement membrane of the corneal endothelium (Figure 3A), suggesting that $\mathrm{Y}-27632$ altered the adhesion properties of the RCECs and up-regulated cell adhesion on the basement membrane in vivo. To ascertain the causal effect of the elevated cell adhesion by Y-27632 in the induction of a pathologically transparent cornea, the histological phenotype of a donor cornea treated with Y-27632 was elucidated using a flat-mount cornea. The expression of ZO- 1 and $\mathrm{Na}^{+} / \mathrm{K}^{+}$-ATPase was evident in RCECs injected with Y-27632, yet it was absent in RCECs injected without Y-27632. RCECs injected with Y-27632 exhibited a monolayer hexagonal cell shape, whereas RCECs injected without Y-27632 exhibited the stratified fibroblastic phenotype. Consistent with the stratified fibroblastic phenotype of RCECs injected without Y-27632, $\alpha$-SMA (a marker of fibroblastic change) was evident in those RCECs (Figure 3B).

The existence of reconstructed corneal endothelium by the injection of RCECs with Y-27632 that expressed Dil, which labels RCECs, indicated that the injected RCECs contributed to the formation of a monolayer of corneal endothelium and to the inducement of corneal transparency (Figure 3B). However, Dil-expressing cells were also observed in the rabbits injected with RCECs without Y-27632, consistent with the results shown in Figure 1C. The presence of Dil-positive cells in the eyes injected with RCECs without Y-27632 may suggest that a limited number of RCECs were able to adhere to the cornea without the assistance of Y-27632, yet changed their phenotype to that of fibroblastic cells. This finding is consistent with those observed in the clinical setting, in which CECs display a fibroblastic phenotype in cases of corneal endothelial dysfunction. ${ }^{22,23}$

\section{ROCK Inhibitor Y-27632 Enhances Cell Adhesion}

To examine the role of the Rho/ROCK signaling pathway in modulating the adhesion properties of primate CECs, cultivated MCECs were plated in combination with ROCK inhibitor Y-27632. Consistent with our previous findings, ${ }^{20}$ phase contrast imaging and actin fiber staining revealed elevated cell adhesion in the Y-27632 treated cells (Figure 4A), and the cell adhesion was enhanced at the conventionally used concentration ${ }^{24}$ (Figure 4B). MCECs treated with Y-27632 showed a markedly improved expression of vinculin in contrast to the nontreated cells (Figure 4C), suggesting that Y-27632 enhanced the cell adhesion via the induction of focal
A
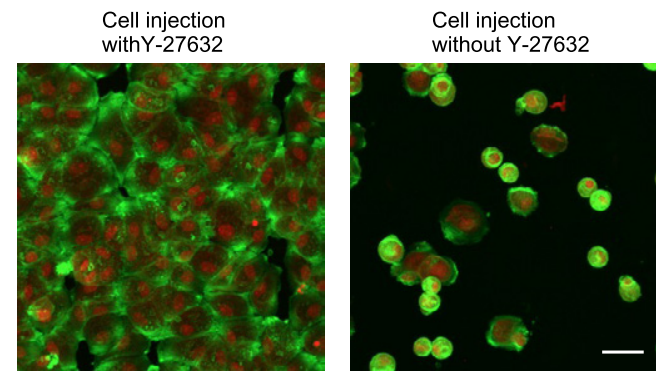

B Cell injection

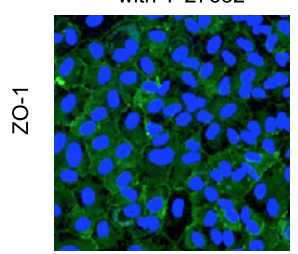

Cell injection without Y-27632
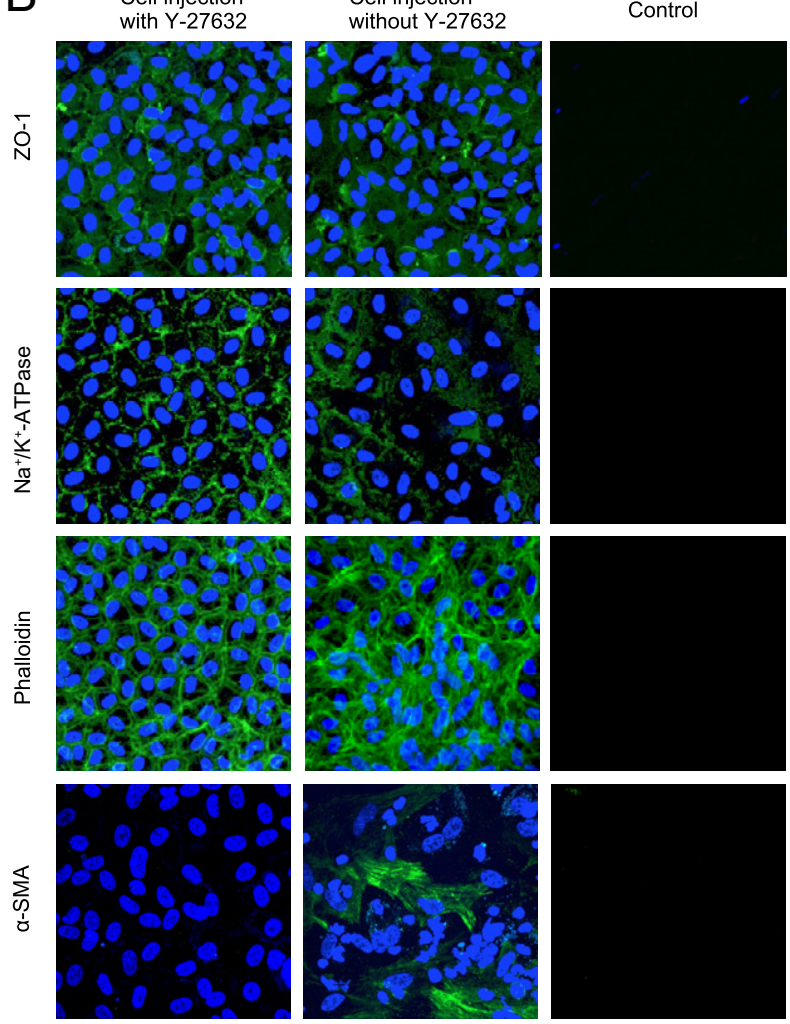

$\overline{\bar{\alpha}}$
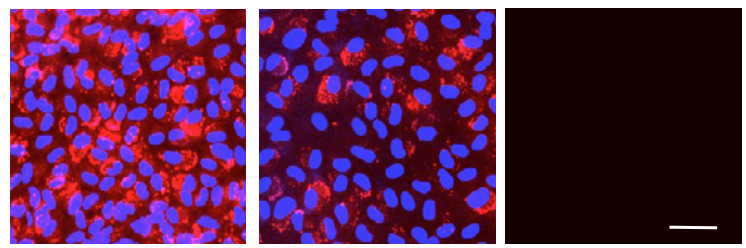

Figure 3. Histological examination of the reconstructed corneal tissue in rabbits after RCEC injection with and without Y-27632. A: Flat-mount examination of the posterior side of the corneal tissue 3 hours after RCEC injection. Green fluorescein shows actin-staining (phalloidin), and red shows nuclear staining by propidium iodide (PI). Scale bar $=100 \mu \mathrm{m}$. B: Histological examination of corneal tissue taken from the rabbit eye 2 weeks after RCEC injection with or without Y-27632. The histological phenotype of the injected RCECs was evaluated by immunofluorescence of $\mathrm{ZO}-1, \mathrm{Na}^{+} / \mathrm{K}^{+}$-ATPase, phalloidin, $\alpha$-SMA, and DiI after 2 weeks. No cells are observed in the control eyes in which the corneal endothelium was scraped. Scale bar $=100 \mu \mathrm{m}$.

adhesion complexes. Considering the interplay between focal adhesion complex molecules and the extracellular matrix, ${ }^{15}$ we next attempted to clarify the effect of Y-27632 on MCEC adhesion onto Descemet's membrane (basement membrane). Consistent with the in vivo experiments shown in Figure 3A, an ex vivo culture system 

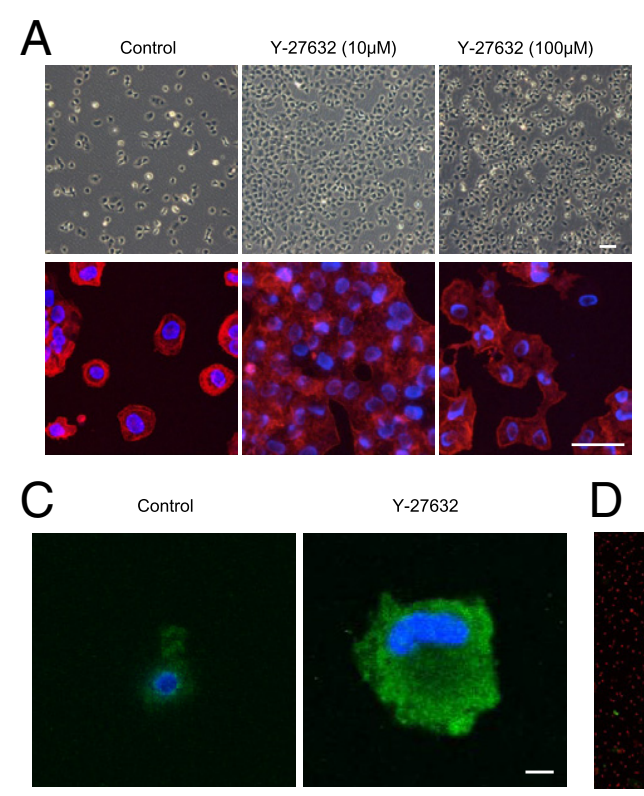

E

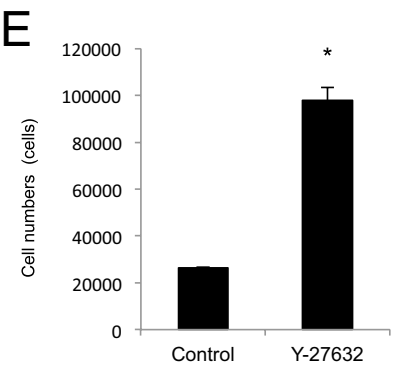

$\mathrm{H}$

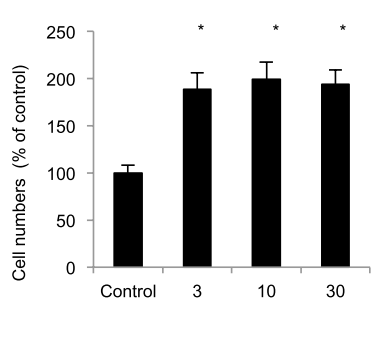

demonstrated that Y-27632 dramatically enhanced the adhesion of the MCECs onto the basement membrane at 3 hours after seeding (Figure 4, D and E).

The direct contribution of the Rho/ROCK signaling pathway in elevating the adhesion properties was elucidated by the knockdown of ROCK I and ROCK II by RNAi (Figure 4, F and G; confirmed with three independent RNAi). Coincidentally, as with the elevated cell adhesion by Y-27632, the knockdown of both ROCK I and ROCK II strongly enhanced the cell adhesion. Because ROCK signaling is necessary to negatively regulate cell adhesion by inhibiting actin depolymerization, we speculate that inhibition of ROCK by Y-27632 promotes actin reorganization, subsequently inducing the enhancement of cell adhesion. In accordance with that speculation, we found that cytochalasin D, which is additionally capable of inhibiting actin polymerization, also elevated the MCEC adhesion (Figure 4H).
Figure 4. Rho/ROCK signaling pathway modulation of the adhesion properties of primate CECs in culture. A: Phase contrast images (top row) and actin fiber staining images (bottom row) reveal elevated cell adhesion in Y-27632treated monkey CECs (MCECs) at 24 hours after seeding on a culture dish. Scale bar $=100 \mu \mathrm{m}$ (top row), $50 \mu \mathrm{m}$ (bottom row). B: The number of adhered MCECs is significantly enhanced by $10 \mu \mathrm{mol} / \mathrm{L}$ and $100 \mu \mathrm{mol} / \mathrm{L}$ of $\mathrm{Y}-27632$ at 24 hours after seeding. Data are expressed as a percentage of the control, mean \pm SEM. ${ }^{*} P<$ 0.01. C: Representative immunofluorescence images of MCECs seeded with or without Y-27632 taken after staining with anti-vinculin at 3 hours after seeding. Scale bar $=10 \mu \mathrm{m}$. D: MCEC were seeded with or without Y-27632 on ex vivo culture system of rabbit Descemet's membrane and stained with actin antibody at 3 hours after seeding. Green fluorescein shows actin staining (phalloidin), and red shows nuclear staining by propidium iodide (PI). Scale bar $=300 \mu \mathrm{m}$. E: MCECs (density: $2.0 \times 10^{5}$ cells) were seeded with or without Y-27632 on rabbit Descemet's membrane, and the number of adhered cells was evaluated at 3 hours after seeding. Data are expressed as mean \pm SEM. ${ }^{*} P<0.01$. F and G: Direct contribution of the Rho/ROCK signaling pathway to the regulation of the adhesion properties of CECs was assessed by the knockdown of ROCK1 (F) or ROCK2 (G). Results are expressed as a percentage of the control, mean \pm SEM. ${ }^{*} P<0.01$. H: MCEC attachment was assessed through the inhibition of actin polymerization by cytochalasin D. Results are expressed as a percentage of the control, mean \pm SEM. ${ }^{*} P<0.01$.

\section{Injection of Cultivated CECs with ROCK Inhibitor Enables Regeneration of Cornea in a Primate Model}

The injection of cultivated MCECs combined with Y-27632 was performed in a cynomolgus monkey in which the corneal endothelium was mechanically removed to produce a pathological dysfunction model. To elucidate the long-term efficacy of the injection of CECs with ROCK inhibitor Y-27632, that monkey model was observed for 3 months. In contrast to the rabbit model, slit-lamp microscopy showed that the monkey eyes injected with MCECs, both with and without Y-27632, exhibited complete corneal transparency within 1 week, and that the transparency persisted throughout 3 months of cell injection with a normal range of thickness $(<600 \mu \mathrm{m})$ (Figure 5A). To evaluate the histological phenotype of donor corneas, two of four mon- 
A

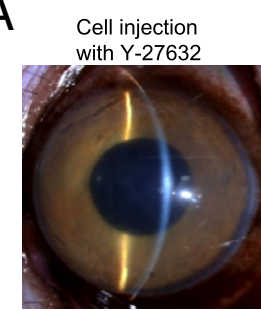

C
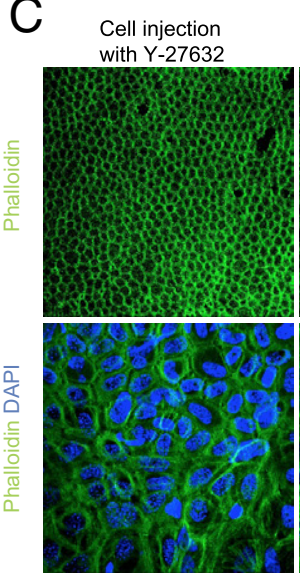

Cell injection
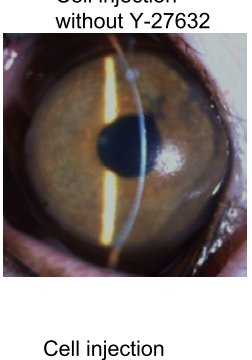
without Y-27632

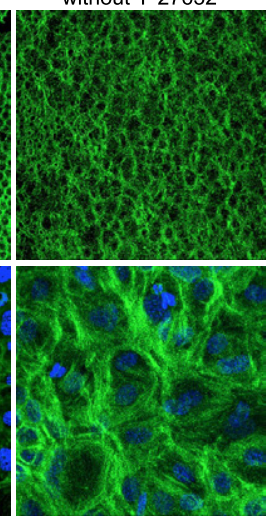

B

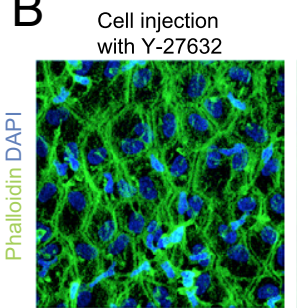

D

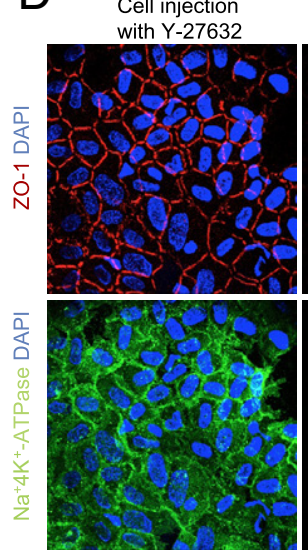

Cell injection

without Y-27632

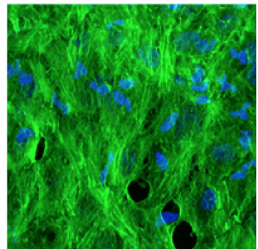

Cell injection without Y-27632

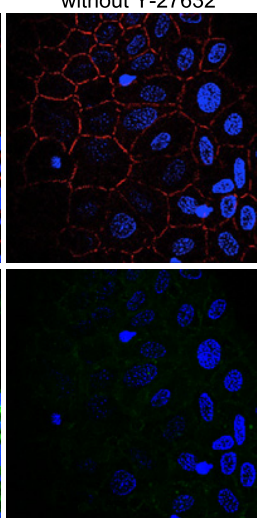

Figure 5. Regeneration of the cornea in a primate model enabled by the injection of cultivated MCECs with Y-27632. A: Monkey eyes injected with MCECs with Y-27632 or without Y-27632 exhibit a transparent cornea with a normal range of thickness $(<600 \mu \mathrm{m})$ after 3 months. B: Histological analysis by actin immunostaining after 2 weeks of cell injection of MCECs injected with or without Y-27632. C: Histological analysis by actin immunostaining after 3 months of cell injection of MCECs injected with or without Y-27632. Top row: lower magnification (green, actin); bottom row: higher magnification (green, actin; blue, DAPI). The cell density of the MCECs injected without Y-27632 was 789 cells $/ \mathrm{mm}^{2}$, whereas that with Y-27632 was 2208 cells $/ \mathrm{mm}^{2}$. D: Histological analysis was performed after immunostaining of ZO-1 (red) or $\mathrm{Na}^{+} / \mathrm{K}^{+}$-ATPase (green). DAPI was used for nuclei staining (blue) keys were euthanized after 2 weeks of MCEC injection and the other two monkeys after 3 months of MCEC injection (one monkey that received MCEC injection with Y-27632 and one monkey that received MCEC injection without Y-27632, respectively, at each of the two time points). It is notable that the MCECs injected with Y-27632 exhibited a monolayer cell shape, whereas the MCECs injected without Y-27632 exhibited a stratified fibroblastic phenotype at the 2-week time point, which mirrored the rabbit model findings (Figure 5B). Although slit-lamp microscopy showed that the monkey eyes injected with MCECs, both with and without Y-27632, exhibited a transparent cornea with a normal-range corneal thickness, MCECs injected without Y-27632 exhibited a fibroblastic phenotype at the cell density of 789 cells/ $\mathrm{mm}^{2}$, whereas MCECs injected with Y-27632 exhibited a monolayer hexagonal cell phenotype at the cell density of 2208 cells $/ \mathrm{mm}^{2}$ (Figure 5C), suggesting that Y-27632 also enhanced the adhesion of MCECs in the in vivo monkey model. Consistent with the rabbit experiments, the MCECs injected with Y-27632 expressed ZO-1 and $\mathrm{Na}^{+} / \mathrm{K}^{+}$-ATPase, yet they were expressed to a lesser degree in the MCECs injected without Y-27632 that exhibited the fibroblastic phenotype (Figure 5D).

\section{Discussion}

Corneal endothelial dysfunction accompanied by visual disturbance is a major indication for corneal transplantation surgery. ${ }^{25}$ Although corneal transplantation is widely performed for corneal endothelial dysfunction, the transplantation of cultivated corneal endothelium is a potential therapeutic strategy. Cultivated CECs derived from older donors have lower proliferative ability, a senescent cell phenotype, and transformed cell morphology, thus suggesting less functional ability than those derived from younger donors. ${ }^{26}$ When corneal endothelium is cultured and stocked as "master cells," it allows for the transplantation of CECs derived from younger donors, thus providing cells with high functional ability and an extended longevity. In addition, it enables an HLA-matching transplantation to reduce the risk of rejection ${ }^{27,28}$ and to overcome the shortage of donor corneas. In the clinical setting, transplantation of corneal endothelium is technically difficult, because it is a monolayer and is located in the anterior chamber. Thus, that anatomical feature led us to hypothesize that the injection of cultivated CECs would be a potent therapy, even though a previous study reported that cell injection itself was ineffective. ${ }^{12}$ The findings of this present study show that the inclusion of ROCK inhibitor Y-27632 elevates the adhesion property of CECs, thus allowing the successful transplantation of CECs to reconstruct functional corneal endothelium damaged by pathological dysfunctions.

We previously reported that the inhibition of ROCK by use of the selective ROCK inhibitor Y-27632 elevates the adhesion of cultured CECs on the substrate, enhances cell proliferation, and suppresses apoptosis. ${ }^{20}$ Although the precise underlying mechanisms have yet to be elucidated, those distinct positive effects of ROCK inhibition enable the establishment of the in vitro expansion of CECs for cultivated corneal endothelial transplantation. ${ }^{20}$ Rho-ROCK signaling carries out a variety of cellular processes such as cell adhesion, morphogenesis, migration, and cell-cycle progression through mediating cytoskeletal dynamics. The Rho GTPase-specific guanine nucleotide exchange factors (Rho GEFs) convert Rho from the guanosine diphosphate (GDP)-bound inactive form to 
the guanosine triphosphate (GTP)-bound active form, thus inducing Rho GTPase activity. Rho regulates a variety of cytoskeletal dynamics that underlie cell morphology and adhesion through the activation of ROCK, a major downstream effector. ${ }^{16,19}$ ROCK signaling modulates acto-myosin contractility through the regulation of myosin phosphorylation and actin dynamics by promoting nucleation and polymerization or by stimulating the severing and depolymerization of existing actin filaments. ${ }^{17,29}$ It is reported that the actin cytoskeleton plays a critical role in regulating the adhesive property through interaction between the actin cytoskeleton and integrin. ${ }^{14,17,18}$ Although the adhesive property is dependent on the cell type and the environmental context, ROCK signaling has been shown to negatively regulate the integrin adhesions of monocytes ${ }^{17}$ and leukocytes. ${ }^{18}$ Findings from a recent study showed that the RhoA-ROCK-PTEN pathway was highly activated when pre-osteoblasts are poorly attached to the substrate, and that the inhibition of this pathway enhances cell adhesion as well as proliferation. ${ }^{30}$ Our findings that the inhibition of ROCK signaling by a selective ROCK inhibitor or by the siRNA enhanced adhesive property of CECs are consistent with the findings of those previous studies. Our findings are also supported by our data that inhibiting actin polymerization by cytochalasin $D$ enhances the adhesive property. Furthermore, we found that vinculin, which is involved in the linkage of the integrin adhesion complex to the actin cytoskeleton, ${ }^{31,32}$ is upregulated in ROCK-inhibitortreated CECs. Further investigation is needed to elucidate whether the ROCK inhibitor promotes the focal adhesions through inhibiting actin polymerization and induces the upregulation of cell adhesion properties on the extracellular matrix (ECM).

Corneal endothelial dysfunctions such as Fuchs's endothelial corneal dystrophy, pseudoexfoliation syndrome, keratitis, and injury induce the fibroblastic transformation of CECs. ${ }^{22,23}$ In addition, CECs reportedly showed fibroblastic transformation during the wound healing process, ${ }^{33}$ and IL-1 $\beta$-mediated FGF-2 produced after an injury reportedly alters CEC morphology and the actin cytoskeleton in a rabbit freezing injury model. ${ }^{34}$ Our findings that RCECs without Y-27632 injected into the anterior chamber of a bullous keratopathy rabbit model exhibited stratified fibroblastic cell morphology and a resultant opaque cornea are consistent with these studies. On the other hand, MCECs without Y-27632 exhibited less fibroblastic phenotype. In our current primate model, a low density of MCECs compensated the pump and barrier functions and resulted in a clear cornea. That finding might possibly be explained by differences in the wound healing process between species. ${ }^{35,36}$ However, because CEC density continuously decreases after keratoplasty, ${ }^{37}$ reconstructed corneal endothelium with Y-27632 at a high cell density is crucial for the successful long-term outcome of transplantation in the clinical setting. To establish the application of a cultivated CEC injection combined with ROCK inhibitor in clinical settings, transplantation models more akin to humans are required, as rabbit CECs exhibit a high proliferative ability in vivo, ${ }^{38}$ unlike human CECs. The findings from this pres- ent study demonstrated that a monkey eye injected with MCECs with Y-27632 exhibited an almost completely clear cornea. Thus, our primate model-based findings suggest that the cell injection therapy in which the cell adhesion is modulated by ROCK inhibitor might prove to be an effective treatment regimen for human corneal endothelial disorders.

In regard to future clinical applications, ROCK inhibitors have been shown to be useful for a wide range of diseases such as cardiovascular disease, pulmonary disease, cancer, and glaucoma. ${ }^{21,39-41}$ Fasudil, one of the ROCK inhibitors, has already been used clinically for the prevention and treatment of cerebral vasospasm, and to date has been therapeutically applied in over 124,000 cases in Japan. ${ }^{21}$ Furthermore, we previously demonstrated that a ROCK inhibitor eye drop enhanced corneal endothelial proliferation in vitro, ${ }^{20}$ as well as in an in vivo animal model, ${ }^{42}$ and it is currently under clinical research for corneal endothelial dysfunction. These facts suggest that the ROCK inhibitor is a therapeutic tool that can be safely and effectively applied in the clinical setting.

In conclusion, the findings of this present study, which are supported by both rabbit and primate corneal endothelial dysfunction models, indicate that ROCK inhibitor Y-27632 will enable the establishment of a cultivatedCEC-based therapy. Modulating actin cytoskeletal dynamics through Rho-ROCK signaling activity serves as a potential for cell-based regenerative medicine to enhance cell engraftment. This novel strategy of using a cell-based therapy combined with a ROCK inhibitor may ultimately provide clinicians with a new therapeutic modality in regenerative medicine, not only for the treatment of corneal endothelial dysfunctions but also for a variety of pathological diseases.

\section{Acknowledgments}

We thank Drs. Yoshiki Sasai and Masatoshi Ohgushi for assistance and invaluable advice regarding ROCK inhibitors, Takahiro Nakagawa for assistance with the monkey experiments, Kenta Yamasaki, Mayumi Yamamoto, Yuri Tsukahara, and Toshie Isobe for technical assistance, and John Bush for reviewing the manuscript.

\section{References}

1. Gorovoy MS: Descemet-stripping automated endothelial keratoplasty. Cornea 2006, 25:886-889

2. Price FW, Jr., Price MO: Descemet's stripping with endothelial keratoplasty in 50 eyes: a refractive neutral corneal transplant. J Refract Surg 2005, 21:339-345

3. Koizumi N, Sakamoto Y, Okumura N, Okahara N, Tsuchiya H, Torii R Cooper LJ, Ban Y, Tanioka H, Kinoshita S: Cultivated corneal endothelial cell sheet transplantation in a primate model. Invest Ophthalmo Vis Sci 2007, 48:4519-4526

4. Koizumi N, Sakamoto Y, Okumura N, Tsuchiya H, Torii R, Cooper LJ, Ban Y, Tanioka H, Kinoshita S: Cultivated corneal endothelial transplantation in a primate: possible future clinical application in corneal endothelial regenerative medicine. Cornea 2008, 27 Suppl 1:S48S55

5. Mimura T, Yamagami S, Yokoo S, Usui T, Tanaka K, Hattori S, Irie S Miyata K, Araie M, Amano S: Cultured human corneal endothelial cell 
transplantation with a collagen sheet in a rabbit model. Invest Ophthalmol Vis Sci 2004, 45:2992-2997

6. Ishino Y, Sano Y, Nakamura T, Connon CJ, Rigby H, Fullwood NJ, Kinoshita S: Amniotic membrane as a carrier for cultivated human corneal endothelial cell transplantation. Invest Ophthalmol Vis Sci 2004, 45:800-806

7. Schachinger V, Erbs S, Elsasser A, Haberbosch W, Hambrecht R, Holschermann H, Yu J, Corti R, Mathey DG, Hamm CW, Suselbeck T, Assmus B, Tonn T, Dimmeler S, Zeiher AM: Intracoronary bone marrow-derived progenitor cells in acute myocardial infarction. $\mathrm{N}$ Engl J Med 2006, 355:1210-1221

8. Tateishi-Yuyama E, Matsubara H, Murohara T, Ikeda U, Shintani $S$, Masaki H, Amano K, Kishimoto Y, Yoshimoto K, Akashi H, Shimada K, Iwasaka T, Imaizumi T: Therapeutic angiogenesis for patients with limb ischaemia by autologous transplantation of bone-marrow cells: a pilot study and a randomised controlled trial. Lancet 2002, 360:427-435

9. Shapiro AM, Lakey JR, Ryan EA, Korbutt GS, Toth E, Warnock GL, Kneteman NM, Rajotte RV: Islet transplantation in seven patients with type 1 diabetes mellitus using a glucocorticoid-free immunosuppressive regimen. N Engl J Med 2000, 343:230-238

10. Yanaga H, Koga M, Imai K, Yanaga K: Clinical application of biotechnically cultured autologous chondrocytes as novel graft material for nasal augmentation. Aesthetic Plast Surg 2004, 28:212-221

11. Mimura T, Shimomura N, Usui T, Noda Y, Kaji Y, Yamgami S, Amano $\mathrm{S}$, Miyata K, Araie M: Magnetic attraction of iron-endocytosed corneal endothelial cells to Descemet's membrane. Exp Eye Res 2003, 76: 745-751

12. Mimura $T$, Yamagami S, Usui T, Ishii $Y$, Ono K, Yokoo S, Funatsu H, Araie M, Amano S: Long-term outcome of iron-endocytosing cultured corneal endothelial cell transplantation with magnetic attraction. Exp Eye Res 2005, 80:149-157

13. Patel SV, Bachman LA, Hann CR, Bahler CK, Fautsch MP: Human corneal endothelial cell transplantation in a human ex vivo model. Invest Ophthalmol Vis Sci 2009, 50:2123-2131

14. Sastry SK, Burridge K: Focal adhesions: a nexus for intracellular signaling and cytoskeletal dynamics. Exp Cell Res 2000, 261:25-36

15. Critchley DR: Focal adhesions-the cytoskeletal connection. Curr Opin Cell Biol 2000, 12:133-139

16. Hall A: Rho GTPases and the actin cytoskeleton. Science 1998 , 279:509-514

17. Worthylake RA, Lemoine S, Watson JM, Burridge K: RhoA is required for monocyte tail retraction during transendothelial migration. J Cell Biol 2001, 154:147-160

18. Worthylake RA, Burridge $\mathrm{K}$ : RhoA and ROCK promote migration by limiting membrane protrusions. J Biol Chem 2003, 278:13578-13584

19. Narumiya S, Tanji M, Ishizaki T: Rho signaling, ROCK and mDia1, in transformation, metastasis and invasion. Cancer Metastasis Rev 2009, 28:65-76

20. Okumura N, Ueno M, Koizumi N, Sakamoto Y, Hirata K, Hamuro J, Kinoshita S: Enhancement on primate corneal endothelial cell survival in vitro by a ROCK inhibitor. Invest Ophthalmol Vis Sci 2009, 50: 3680-3687

21. Liao JK, Seto M, Noma K: Rho kinase (ROCK) inhibitors. J Cardiovasc Pharmacol 2007, 50:17-24

22. Naumann GO, Schlotzer-Schrehardt U: Keratopathy in pseudoexfoliation syndrome as a cause of corneal endothelial decompensation: a clinicopathologic study. Ophthalmology 2000, 107:1111-1124

23. Kawaguchi R, Saika S, Wakayama M, Ooshima A, Ohnishi Y, Yabe H: Extracellular matrix components in a case of retrocorneal membrane associated with syphilitic interstitial keratitis. Cornea 2001, 20:100-103
24. Narumiya S, Ishizaki T, Uehata M: Use and properties of ROCKspecific inhibitor Y-27632. Methods Enzymol 2000, 325:273-284

25. Darlington JK, Adrean SD, Schwab IR: Trends of penetrating keratoplasty in the United States from 1980 to 2004. Ophthalmology 2006, 113:2171-2175

26. Joyce NC, Zhu CC: Human corneal endothelial cell proliferation: potential for use in regenerative medicine. Cornea 2004, 23:S8-S19

27. Khaireddin R, Wachtlin J, Hopfenmuller W, Hoffmann F: HLA-A HLA-B and HLA-DR matching reduces the rate of corneal allograft rejection. Graefes Arch Clin Exp Ophthalmol 2003, 241:1020-1028

28. Coster DJ, Williams KA: The impact of corneal allograft rejection on the long-term outcome of corneal transplantation. Am J Ophthalmol 2005, 140:1112-1122

29. Ishizaki T, Uehata M, Tamechika I, Keel J, Nonomura K, Maekawa M, Narumiya S: Pharmacological properties of Y-27632, a specific inhibitor of rho-associated kinases. Mol Pharmacol 2000, 57:976-983

30. Yang S, Kim HM: The RhoA-ROCK-PTEN pathway as a molecular switch for anchorage dependent cell behavior. Biomaterials 2012, 33:2902-2915

31. Geiger B: A $130 \mathrm{~K}$ protein from chicken gizzard: its localization at the termini of microfilament bundles in cultured chicken cells. Cell 1979, 18:193-205

32. Burridge K, Feramisco JR: Microinjection and localization of a $130 \mathrm{~K}$ protein in living fibroblasts: a relationship to actin and fibronectin. Cell 1980, 19:587-595

33. Ichijima H, Petroll WM, Jester JV, Barry PA, Andrews PM, Dai M, Cavanagh HD: In vivo confocal microscopic studies of endothelial wound healing in rabbit cornea. Cornea 1993, 12:369-378

34. Lee HT, Lee JG, Na M, Kay EP: FGF-2 induced by interleukin-1 beta through the action of phosphatidylinositol 3-kinase mediates endothelial mesenchymal transformation in corneal endothelial cells. J Biol Chem 2004, 279:32325-32332

35. Matsubara M, Tanishima T: Wound-healing of the corneal endothelium in the monkey: a morphometric study. Jpn J Ophthalmol 1982, 26:264-273

36. Matsubara M, Tanishima T: Wound-healing of corneal endothelium in monkey: an autoradiographic study. Jpn J Ophthalmol 1983, 27:444450

37. Bourne WM, Nelson LR, Hodge DO: Central corneal endothelial cel changes over a ten-year period. Invest Ophthalmol Vis Sci 1997, 38:779-782

38. Van Horn DL, Sendele DD, Seideman S, Buco PJ: Regenerative capacity of the corneal endothelium in rabbit and cat. Invest Ophthalmol Vis Sci 1977, 16:597-613

39. Koga T, Awai M, Tsutsui J, Yue BY, Tanihara H: Rho-associated protein kinase inhibitor, Y-27632, induces alterations in adhesion, contraction and motility in cultured human trabecular meshwork cells. Exp Eye Res 2006, 82:362-370

40. Olson MF: Applications for ROCK kinase inhibition. Curr Opin Cell Biol 2008, 20:242-248

41. Tokushige $\mathrm{H}$, Inatani M, Nemoto S, Sakaki H, Katayama K, Uehata M, Tanihara H: Effects of topical administration of $\mathrm{y}-39983$, a selective rho-associated protein kinase inhibitor, on ocular tissues in rabbits and monkeys. Invest Ophthalmol Vis Sci 2007, 48:3216-3222

42. Okumura N, Koizumi N, Ueno M, Sakamoto Y, Takahashi H, Hirata K, Torii R, Hamuro J, Kinoshita S: Enhancement of corneal endothelium wound healing by Rho-associated kinase (ROCK) inhibitor eye drops. Br J Ophthalmol 2011, 95:1006-1009 